DEPÓSITO LEGAL ZU2020000153

Esta publicación científica en formato digital

es continuidad de la revista impresa

ISSN 0041-8811

E-ISSN 2665-0428

Revista

de la

Universidad

del Tunlia

Fundada en 1947

por el Dr. Jesús Emrique Lossada

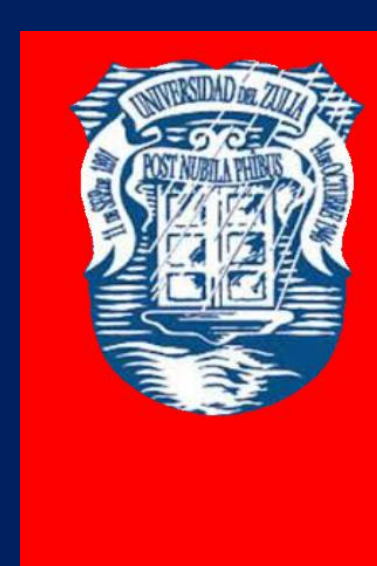

Ciencias

Sociales

y Arte

Aกัต 11 No 31

Septiembre - Diciembre 2021

Tercera ípoca

Maracailbo-Venezuela 
REVISTA DE LA UNIVERSIDAD DEL ZULIA. 3épeca. Año 11 N³1, 2020 José Walter Coronel Chugden et al /// Clima organizacional, satisfacción laboral... 52-70

DOI: http://dx.doi.org/10.46925//rdluz.31.05

\title{
Clima organizacional, satisfacción laboral y gestión administrativa en los órganos de apoyo de una universidad pública
}

\author{
José Walter Coronel Chugden* \\ Guido Ayay Arista** \\ Manuel Emilio Milla Pino***
}

RESUMEN

La investigación tuvo como objetivo determinar la correlación entre el clima organizacional, satisfacción laboral y gestión administrativa en los órganos de apoyo de una universidad pública al norte de Perú, 2019; en el estudio se empleó el diseño descriptivo correlacional. La muestra estuvo conformada por 86 miembros del personal administrativo. La técnica fue la encuesta y como instrumentos se aplicó tres cuestionarios con índices de fiabilidad: [0.929], [0.947] y $[0,862]$ respectivamente. Los resultados evidencian un clima organizacional de nivel alto (proporción=79\%), la satisfacción laboral de nivel satisfecho (proporción=66\%) y la gestión administrativa de nivel competente (proporción=73\%). Así mismo, existe correlación moderada positiva entre par de variables, clima organizacional y satisfacción laboral (Rs=+0.290 moderada, $\mathrm{p}=0.007<0.01$ ) clima organizacional y gestión administrativa $(\mathrm{Rs}=+0.305$ moderada, $\mathrm{p}=0.004<0.01)$ satisfacción laboral y gestión administrativa $(\mathrm{Rs}=+0.347$ moderada, $\mathrm{p}=0.001<0.01$ ). En conclusión, existe una correlación positiva de nivel moderada entre clima organizacional, satisfacción laboral y gestión administrativa (Correlación $R s=+0.408, \mathrm{p}=0.001<0.05)$.

PALABRAS CLAVE: clima organizacional, satisfacción laboral y gestión administrativa.

*Universidad Nacional Toribio Rodríguez de Mendoza de Amazonas, Perú, http://orcid.org/0000-0002-7031-0755, prof_walter_jose@hotmail.com

**Universidad Nacional Toribio Rodríguez de Mendoza de Amazonas, Perú, https://orcid.org/0000-0002-5716-7376, estudioaprendizaje@gmail.com

***Universidad Nacional de Jaén, Perú, https://orcid.org/0000-0003-3931-9804, memilla22@yahoo.com.mx

Recibido: 01/07/2020

Aceptado: 05/09/2020 
REVISTA DE LA UNIVERSIDAD DEL ZULIA. 3é época. Año 11 N³1, 2020

José Walter Coronel Chugden et al /// Clima organizacional, satisfacción laboral... 52-70

DOI: http://dx.doi.org/10.46925//rdluz.31.05

\section{Organizational climate, job satisfaction and administrative management in the support bodies of a public university}

ABSTRACT

The research aimed to determine the correlation between organizational climate, job satisfaction and administrative management in the support bodies of a public university in northern Peru, 2019; the study used the correlated descriptive design. The sample consisted of 86 administrative staff members. The technique was the survey and as instruments three questionnaires were applied with reliability rates: [0.929], [0.947] and [0.862] respectively. The results show a high-level organizational climate (proportion 79\%), satisfied level job satisfaction (proportion 66\%) and the competent-level administrative management (proportion $73 \%$ ). There is also a positive moderate correlation between a pair of variables, organizational climate and job satisfaction (moderate Rs+0.290, p-0.007<0.01) organizational climate and administrative management (moderate Rs-+0.305, p-0.004<0.01) occupational satisfaction and administrative management (Rs-+0.347 moderate, p-0.001<lt 0.01). In conclusion, there is a positive correlation of moderate level between organizational climate, job satisfaction and administrative management (Correlation Rs-+0.408, p-0.001<0.05).

KEYWORDS: organizational climate, job satisfaction and administrative management.

Introducción

El clima organizacional, viene a ser el conjunto de particularidades que definen el ambiente de trabajo en una institución cuyo efecto repercute en el comportamiento, las relaciones y las cualidades de los trabajadores (Bernal et al., 2015); es un factor fundamentalmente variable y de mediano plazo (Bustamante-Ubilla et al., 2015). En ese contexto, estudiar el clima organizacional, permite entender la conducta humana en las instituciones (Pecino-Medina et al., 2017; Peña et al., 2015). No obstante, los estudios sobre el clima organizacional son limitados y han utilizado diseños puramente observacionales dificultando determinar la dirección de la causalidad (Falkenström et al., 2018).

Por otra parte, las investigaciones sobre el clima organizacional a nivel de unidades de formación superior, también son limitadas, a pesar de que en estas existe un gran potencial de recurso humano. En tal sentido, en las universidades se observa el aumento del número estudiantes y de personal administrativo, sin embargo, tal aumento no es proporcional, lo cual ejerce influencia en el clima organizacional y en la satisfacción en el trabajo (Adeniji et 
REVISTA DE LA UNIVERSIDAD DEL ZULIA. 3é época. Año 11 N³1, 2020 José Walter Coronel Chugden et al /// Clima organizacional, satisfacción laboral... 52-70

DOI: $\underline{\text { http://dx.doi.org/10.46925//rdluz.31.05 }}$

al., 2018) y en algunos casos el efecto es negativo, tal como se reportan crecientes índices de violencia en el trabajo a nivel institucional (J. Hernández et al., 2013).

En contraste, el clima organizacional mejora si el involucramiento de todos los sectores forma parte de una cultura que fomenta el compromiso, la creatividad, el entusiasmo y el trabajo en equipo (Bernal et al., 2015; García-Guiu et al., 2014; Mendoza Deza et al, 2020). De tal modo, el apoyo organizacional en una institución, influye indirecta y de manera positiva en el empoderamiento profesional y en la realización efectiva de las tareas del puesto de trabajo (Román-Calderón et al., 2016).

Por otro lado, en referencias a la satisfacción laboral, Weiss (2002) afirma que es una actitud; de modo que, la satisfacción laboral, es el efecto emocional de si el puesto cumple o no con las aspiraciones del personal que labora (Merino-Plaza et al., 2018). En tal sentido, la satisfacción laboral está sujeta a representar los sentimientos, así como las actitudes positivas hacia la ocupación de algo (Kohantorabi y Abolmaali, 2014). Para Ortiz et al., (2017) la satisfacción laboral es el precedente de la conducta humana, relacionado con la productividad.

Sin embargo, el grado de satisfacción laboral del personal que trabaja para un Estado en el sector público o privado, es un tema que no se deja de lado en la administración actual, puesto que, el logro de objetivos definidos depende de esta (Tinoco et al., 2014). En consecuencia, la satisfacción de los miembros del equipo demanda cooperación y comunicación, metas y compromisos comunes, roles y objetivos claros para apoyar el trabajo colaborativo y la figura de un líder transformador con la finalidad de fortificar el clima, la plática y la inventiva (Espinoza et al., 2018).

En relación a la problemática expuesta, numerosos estudios indican que los trabajadores expuestos a condiciones inadecuadas de trabajo, empleo inestable, bajos salarios y beneficios, deficiente salud y seguridad, tienen frustración e insatisfacción con bajos niveles de desempeño y productividad (Navarro, 2012), conllevando a obtener un alto grado de estrés, que se concibe como un peligro para la salud del personal que labora (Aguirre et al., 2015; Azanza et al., 2015; Cajas et al., 2017; Lima et al., 2013; Plascencia-Campos et al., 2019; Vallellano y Rubio-Valdehita, 2018). En tal sentido, la satisfacción laboral, mejora si se apoya del capital psicológico (Gómez-Perdomo et al., 2017). En tanto, los administradores deberían 
REVISTA DE LA UNIVERSIDAD DEL ZULIA. 3é época. Año 11 N³1, 2020 José Walter Coronel Chugden et al /// Clima organizacional, satisfacción laboral... 52-70

DOI: $\underline{\text { http://dx.doi.org/10.46925//rdluz.31.05 }}$

adoptar medidas de intervención para optimizar la satisfacción del trabajo y certificar una atención óptima (Yew et al., 2018).

En otro orden de ideas, la gestión administrativa, es una sucesión estratégica combinada de funciones como: planeación, organización, dirección y control (Castillón, 2014; Menoza-Briones, 2017). La administración visualiza el ingreso, la elaboración y administración del presupuesto, el financiamiento y la utilización de indicadores (Palma, 2016). En efecto si se da una adecuada integración de la institución con el contexto, sobre la base de una mejora continua, se puede lograr un progreso importante (Gónzales et al., 2014; Hernández et al., 2017). Para que ello ocurra, el personal directivo es responsable y principal encargado de la gestión administrativa (Riffo, 2019). En contraste, son pocas las investigaciones realizadas en este tema, que permita aportar conocimiento necesario para beneficio en la toma de decisiones directivas (Díaz et al., 2014). Por ello, el argumento concluyente y heurístico pone a discusión una «verdad», de cuál diseño administrativo es adecuado, el de Taylor, Ford, Weber, Ohno, Elton, Fiedler, Ludwig Von, o de Chiavenato (Galicia y Monroy, 2016).

En tal contexto, hacer frente a los nuevos cambios que el mundo exige, obliga a las instituciones a mejorar en eficacia y eficiencia; sin embargo, a partir de la organización, los diversos instrumentos que sobrellevan la gestión centran sus objetivos en los resultados, dejando de lado los procesos (Martínez-Corona et al., 2020). Por consiguiente, los problemas de coordinación administrativa, la insuficiencia de estímulos asertivos y las rigideces de nuestras organizaciones, son limitaciones que determinan la transformación de los sistemas (Palma, 2016).

En consecuencia, en la región de Amazonas, concretamente en la Universidad Nacional Toribio Rodríguez de Mendoza, como universidad pública, existen problemas, tanto laborales, académicos y administrativos. Por su parte, (Santillán, 2015) encontró que la Universidad no le presta real importancia al clima organizacional. A ello, Magallán (2018) y (Terrones, 2017) añade que son los factores intrínsecos y extrínsecos que causan efecto en la mejora de los servicios de la educación universitaria.

Los hechos descritos, conllevaron a proyectar una investigación descriptivacorrelacional, guiado por el siguiente problema de investigación ¿Cuál es la correlación entre el clima organizacional, satisfacción laboral y la gestión administrativa en los órganos de 
REVISTA DE LA UNIVERSIDAD DEL ZULIA. 3é época. Año 11 N³1, 2020 José Walter Coronel Chugden et al /// Clima organizacional, satisfacción laboral... 52-70

DOI: $\underline{\text { http://dx.doi.org/10.46925//rdluz.31.05 }}$

apoyo de la Universidad Nacional Toribio Rodríguez de Mendoza de Amazonas, 2019? Para ello, como objetivo general se propuso determinar la correlación entre el clima organizacional, satisfacción laboral y gestión administrativa y como objetivos específicos se propuso estimar la asociación entre el clima organizacional y satisfacción laboral, estimar la asociación entre el clima organizacional y gestión administrativa y finalmente, estimar el grado de asociación entre satisfacción laboral y gestión administrativa.

1. Material y métodos

1.1. Población, muestra y muestreo

La población estuvo conformada por 96 miembros del personal administrativo de la Universidad Nacional Toribio Rodríguez de Mendoza de Amazonas (UNTRM), 2019.

La muestra tuvo un tamaño de 86 , que se traduce en el 89,58 \% de la población, lo que garantiza una cantidad adecuada en función de la representatividad. Esta muestra fue seleccionada siguiendo las pautas establecidas para un muestreo estratificado con afijación proporcional, con el propósito de que todos los sectores que conforman el personal administrativo estén debidamente representados, lo que indica que la muestra estuvo conformada por trabajadores administrativos cuya condición laboral es contratado o nombrado.

\subsection{Diseño de la investigación}

La investigación fue de tipo descriptiva y empleó un diseño correlacional (Sánchez y Reyes, 2017) que permitió determinar el grado de relación entre las variables de estudio.

\subsection{Métodos, técnicas e instrumentos}

El método científico y sistémico priorizan en la investigación, así como el deductivo e inductivo; la interpretación de su correlato ha sido el análisis conceptual cuantitativo preeminente.

Como técnica se utilizó la encuesta y como instrumentos se aplicaron tres cuestionarios. Los cuestionarios que evaluaron el clima organizacional y la satisfacción laboral fueron obtenidos de Chiang, et al. (2008) y el cuestionario sobre gestión administrativa fue tomado de Domínguez (2017), todos válidos y confiables estadísticamente, 
REVISTA DE LA UNIVERSIDAD DEL ZULIA. 3é época. Año 11 N³1, 2020 José Walter Coronel Chugden et al /// Clima organizacional, satisfacción laboral... 52-70

DOI: $\underline{\text { http://dx.doi.org/10.46925//rdluz.31.05 }}$

cuyos índices de fiabilidad son: [0.929], [0.947] y [0,862] respectivamente. Los cuestionarios constan de 40, 37 y 32 ítems respectivamente, con una escala de respuesta entre: l=Totalmente en desacuerdo y 5 Muy de acuerdo=5.

La información recopilada en la presente investigación permitió la elaboración de una matriz de datos, la cual fue procesada aplicando el coeficiente de correlación ordinal de rangos de Spearman, dada la escala ordinal de medición de las variables, se construyeron tablas de distribución de frecuencias para un posterior análisis porcentual y se realizaron gráficos con el objeto de visualizar el comportamiento de las variables. El software utilizado fue el SPSS V. 25.

2. Resultados

Tabla 1. Distribución de las variables según niveles en los órganos de apoyo de una universidad pública

\begin{tabular}{cccc}
\hline Variables & Niveles & Trabajadores & Porcentaje \\
\hline \multirow{4}{*}{ Clima } & Muy bajo & 4 & $5 \%$ \\
organizacional & Bajo & 10 & $12 \%$ \\
& Alto & 65 & $76 \%$ \\
& Muy alto & 7 & $8 \%$ \\
\cline { 2 - 4 } & Total & 86 & $100 \%$ \\
\hline \multirow{5}{*}{ Satisfacción laboral } & Nada & & \\
& satisfactorio & 3 & $3 \%$ \\
& Poco & & \\
& satisfactorio & 1 & $1 \%$ \\
& No seguro & 24 & $28 \%$ \\
& Satisfecho & 57 & $66 \%$ \\
& Muy satisfecho & 1 & $1 \%$ \\
\cline { 2 - 4 } & Total & 86 & $100 \%$ \\
Gestión & Deficiente & 2 & $2 \%$ \\
Administrativa & Satisfactorio & 15 & $17 \%$ \\
& Competente & 63 & $73 \%$ \\
& Destacado & 6 & $7 \%$ \\
\cline { 2 - 4 } & Total & 86 & $100 \%$ \\
\hline
\end{tabular}

Fuente: Elaboración según encuesta aplicada.

En la tabla y figura 1 , se evidencia que el nivel de clima organizacional en los trabajadores de los órganos de apoyo de la Universidad evaluada es de 5\% muy bajo, 12\% bajo, $76 \%$ alto y $8 \%$ muy alto, lo cual significa que la mayor proporción de trabajadores tienen una 
REVISTA DE LA UNIVERSIDAD DEL ZULIA. 3é época. Año 11 N³1, 2020 José Walter Coronel Chugden et al /// Clima organizacional, satisfacción laboral... 52-70

DOI: $\underline{\text { http://dx.doi.org/10.46925//rdluz.31.05 }}$

percepción de nivel alto en el clima organizacional. De igual manera, se evidencia que el nivel de satisfacción laboral en los trabajadores de los órganos de apoyo de la Universidad es del $3 \%$ nada satisfecho, $1 \%$ poco satisfecho, $28 \%$ no seguro, $66 \%$ satisfecho y $1 \%$ muy satisfecho, lo cual significa que la mayor proporción de trabajadores tienen una percepción de nivel satisfecho. Así también, En la tabla 3, se evidencia que el nivel de gestión administrativa en los trabajadores de los órganos de apoyo de la UNTRM es: 2\% deficiente, 17\% satisfactorio, 73\% competente y 7\% destacado, lo cual significa que la mayor proporción de trabajadores tienen una percepción de nivel competente en la gestión administrativa.

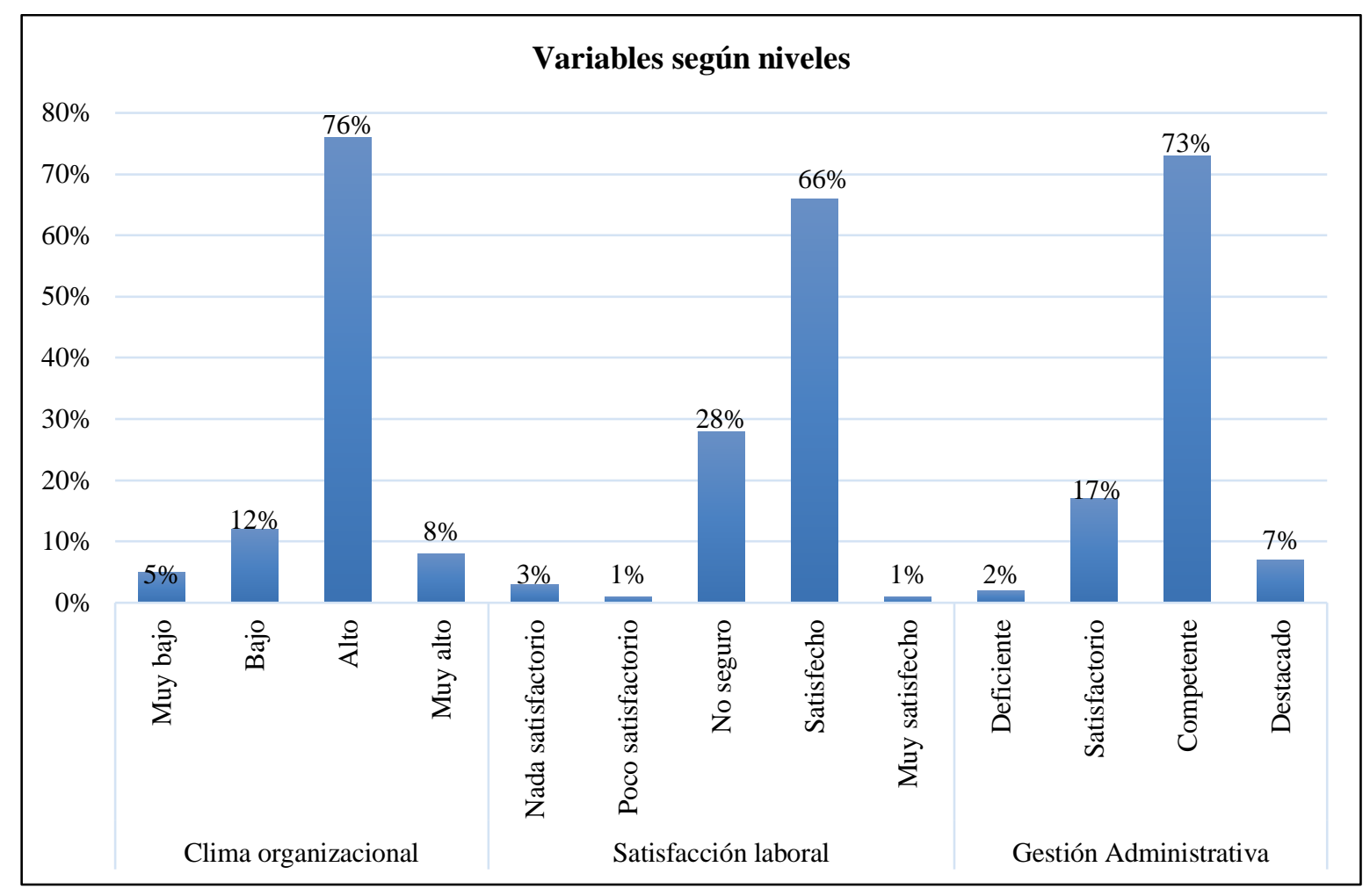

Figura 1. Distribución de las variables según niveles en los órganos de apoyo de una universidad pública

En la tabla 2 se analiza el nivel de las 8 dimensiones del clima organizacional; así, en la dimensión innovación, se encontró: muy bajo 10\%, bajo 12\%, alto 69\%, muy alto 9\%; para la dimensión equidad: muy bajo 9\%, bajo 15\%, alto 60\%, muy alto 15\%; para la dimensión reconocimiento: muy bajo $7 \%$, bajo $21 \%$, alto 69\%, muy alto 3\%; para la dimensión apoyo: muy bajo 9\%, bajo 5\%, alto 60\%, muy alto 26\%; para la dimensión presión: muy bajo 6\%, bajo 57\%, 
REVISTA DE LA UNIVERSIDAD DEL ZULIA. 3é época. Año 11 N³1, 2020 José Walter Coronel Chugden et al /// Clima organizacional, satisfacción laboral... 52-70

DOI: $\underline{\text { http://dx.doi.org/10.46925//rdluz.31.05 }}$

alto 37\%, muy alto 0\%; para la dimensión confianza: muy bajo 7\%, bajo 7\%, alto 52\%, muy alto 34\%; para la dimensión cohesión: muy bajo 6\%, bajo 30\%, alto 53\%, muy alto 10\%; para la dimensión autonomía: muy bajo 0\%, bajo 22\%, alto 66\%, muy alto 12\%. Por lo que se determina que, de las 8 dimensiones, 7 de ellas tienen en mayor proporción un nivel alto, sin embargo, la dimensión presión con un nivel bajo en mayor proporción.

Tabla 2. Distribución del nivel de las dimensiones del clima organizacional

\begin{tabular}{lcccccc}
\hline \multirow{2}{*}{ Dimensiones } & \multicolumn{6}{c}{ Niveles de dimensiones del clima organizacional } \\
& Cantidad & Muy bajo & Bajo & Alto & Muy alto & Total \\
\hline \multirow{2}{*}{ Autonomía } & Trabajadores & 0 & 19 & 57 & 10 & 86 \\
& $\%$ & $0 \%$ & $22 \%$ & $66 \%$ & $12 \%$ & $100 \%$ \\
\hline \multirow{2}{*}{ Cohesión } & Trabajadores & 5 & 26 & 46 & 9 & 86 \\
& $\%$ & $6 \%$ & $30 \%$ & $53 \%$ & $10 \%$ & $100 \%$ \\
\hline \multirow{2}{*}{ Confianza } & Trabajadores & 6 & 6 & 45 & 29 & 86 \\
& $\%$ & $7 \%$ & $7 \%$ & $52 \%$ & $34 \%$ & $100 \%$ \\
\hline \multirow{2}{*}{ Presión } & Trabajadores & 5 & 49 & 32 & 0 & 86 \\
& $\%$ & $6 \%$ & $57 \%$ & $37 \%$ & $0 \%$ & $100 \%$ \\
\hline \multirow{2}{*}{ Apoyo } & Trabajadores & 8 & 4 & 52 & 22 & 86 \\
& $\%$ & $9 \%$ & $5 \%$ & $60 \%$ & $26 \%$ & $100 \%$ \\
\hline \multirow{2}{*}{ Reconocimiento } & Trabajadores & 6 & 18 & 59 & 3 & 86 \\
& $\%$ & $7 \%$ & $21 \%$ & $69 \%$ & $3 \%$ & $100 \%$ \\
\hline \multirow{2}{*}{ Equidad } & Trabajadores & 8 & 13 & 52 & 13 & 86 \\
& $\%$ & $9 \%$ & $15 \%$ & $60 \%$ & $15 \%$ & $100 \%$ \\
\hline \multirow{2}{*}{ Innovación } & Trabajadores & 9 & 10 & 59 & 8 & 86 \\
& $\%$ & $10 \%$ & $12 \%$ & $69 \%$ & $9 \%$ & $100 \%$ \\
\hline
\end{tabular}

Fuente: Elaboración según encuesta aplicada.

En función a los resultados descritos, se pudo evaluar los puntajes promedios en una escala Likert [1-5] de las dimensiones de la variable clima organizacional, donde se estableció el orden de priorización para mejorar tal variable, donde el puntaje ideal en escala ordinal fue de 5 puntos. Es por ello, que para mejorar el clima organizacional, en la dimensión presión debe darse como prioridad 1, media=2.95; reconocimiento (prioridad 2, media=3.26); cohesión (prioridad 3, media=3.32); equidad (prioridad 4, media=3.40); innovación (prioridad 5, media=3.44); autonomía (prioridad 6, media=3.53); apoyo (prioridad 7, media=3.67) y confianza (prioridad 8, media=3.77) y en general un puntaje promedio de 3.42 puntos de 5 puntos como lo ideal para mejorar la variable clima organizacional. 
REVISTA DE LA UNIVERSIDAD DEL ZULIA. 3épeca. Año 11 N³1, 2020 José Walter Coronel Chugden et al /// Clima organizacional, satisfacción laboral... 52-70

DOI: $\underline{\text { http://dx.doi.org/10.46925//rdluz.31.05 }}$

En la tabla 3, se presenta el nivel de las 6 dimensiones de la satisfacción laboral, por lo que en la dimensión satisfacción por el trabajo en general, se evidenció: nada satisfecho 4\%, $5 \%$ poco satisfecho, $20 \%$ no seguro, $6 \%$ satisfecho, $5 \%$ muy satisfecho; para la dimensión satisfacción por el ambiente físico del trabajo: nada satisfecho 1\%, 2\% poco satisfecho, $23 \%$ no seguro, 58\% satisfecho, 16\% muy satisfecho; para la dimensión satisfacción por la forma en que realiza su trabajo: nada satisfecho 5\%, 5\% poco satisfecho, 13\% no seguro, 67\% satisfecho, 10\% muy satisfecho; para la dimensión satisfacción por las oportunidades de desarrollo: nada satisfecho 5\%, 12\% poco satisfecho, $31 \%$ no seguro, $51 \%$ satisfecho, $1 \%$ muy satisfecho; para la dimensión satisfacción por la relación subordinado supervisor: nada satisfecho $1 \%, 19 \%$ poco satisfecho, $26 \%$ no seguro, $52 \%$ satisfecho, $2 \%$ muy satisfecho; para la dimensión satisfacción por la remuneración: nada satisfecho 4\%, 40\% poco satisfecho, $36 \%$ no seguro, 19\% satisfecho, 1\% muy satisfecho. Por lo que se determina que de las 6 dimensiones 5 de ellas tienen en mayor proporción de un nivel satisfecho, sin embargo, la dimensión satisfacción por la remuneración con un nivel poco satisfecho en mayor proporción.

En tal sentido, para mejorar la satisfacción laboral, se evaluó el orden de prioridad según sus dimensiones. Así por ejemplo, es la dimensión satisfacción con la remuneración la que debe atenderse con prioridad 1, media=3.03; satisfacción con las oportunidades de desarrollo (prioridad 2, media=3.31), satisfacción con la relación subordinado-supervisor (prioridad 3, media=3.42), satisfacción por el trabajo general (prioridad 4, media=3.51), satisfacción con la forma en que realiza el trabajo (prioridad 5, media=3.66), satisfacción por el ambiente físico del trabajo (prioridad 6, media=3.82) y en general para mejorar la satisfacción laboral se tiene un puntaje promedio de 3.51 puntos de un puntaje ideal de 5 puntos. 
REVISTA DE LA UNIVERSIDAD DEL ZULIA. 3 3 época. Año 11 N³1, 2020 José Walter Coronel Chugden et al /// Clima organizacional, satisfacción laboral... 52-70

DOI: http://dx.doi.org/10.46925//rdluz.31.05

Tabla 3. Distribución del nivel de las dimensiones de la satisfacción laboral

\begin{tabular}{|c|c|c|c|c|c|c|c|}
\hline \multirow[b]{2}{*}{ Dimensiones } & \multicolumn{6}{|c|}{ Niveles de dimensiones de la satisfacción laboral } & \multirow[b]{2}{*}{ Total } \\
\hline & Cantidad & $\begin{array}{c}\text { Nada } \\
\text { satisfech } \\
\text { o }\end{array}$ & $\begin{array}{c}\text { Poco } \\
\text { satisfech } \\
\text { o } \\
\end{array}$ & $\begin{array}{l}\text { No } \\
\text { seguro }\end{array}$ & $\begin{array}{c}\text { Satisfech } \\
\text { o }\end{array}$ & $\begin{array}{c}\text { Muy } \\
\text { satisfech } \\
\text { o }\end{array}$ & \\
\hline Satisfacción por & Trabajadores & 3 & 4 & 17 & 58 & 4 & 86 \\
\hline general & $\%$ & $4 \%$ & $5 \%$ & $20 \%$ & $66 \%$ & $5 \%$ & $100 \%$ \\
\hline Satisfacción en el & Trabajadores & 1 & 2 & 20 & 50 & 13 & 86 \\
\hline $\begin{array}{l}\text { ambiente físico } \\
\text { del trabajo }\end{array}$ & $\%$ & $1 \%$ & $2 \%$ & $23 \%$ & $58 \%$ & $16 \%$ & $100 \%$ \\
\hline Satisfacción por & Trabajadores & 4 & 4 & 11 & 58 & 9 & 86 \\
\hline $\begin{array}{l}\text { la forma en que } \\
\text { realiza su trabajo }\end{array}$ & $\%$ & $5 \%$ & $5 \%$ & $13 \%$ & $67 \%$ & $10 \%$ & $100 \%$ \\
\hline $\begin{array}{l}\text { Satisfacción por } \\
\text { las }\end{array}$ & Trabajadores & 4 & 10 & 27 & 44 & 1 & 86 \\
\hline $\begin{array}{l}\text { oportunidades de } \\
\text { desarrollo }\end{array}$ & $\%$ & $5 \%$ & $12 \%$ & $31 \%$ & $51 \%$ & $1 \%$ & $100 \%$ \\
\hline $\begin{array}{l}\text { Satisfacción por } \\
\text { la relación }\end{array}$ & Trabajadores & 1 & 16 & 22 & 45 & 2 & 86 \\
\hline $\begin{array}{l}\text { subordinado } \\
\text { supervisor }\end{array}$ & $\%$ & $1 \%$ & $19 \%$ & $26 \%$ & $52 \%$ & $2 \%$ & $100 \%$ \\
\hline $\begin{array}{l}\text { Satisfacción por } \\
\text { la remuneración }\end{array}$ & $\begin{array}{c}\text { Trabajadores } \\
\%\end{array}$ & $\begin{array}{c}3 \\
4 \%\end{array}$ & $\begin{array}{c}35 \\
40 \%\end{array}$ & $\begin{array}{c}31 \\
36 \%\end{array}$ & $\begin{array}{c}16 \\
19 \%\end{array}$ & $\begin{array}{c}1 \\
1 \%\end{array}$ & $\begin{array}{r}86 \\
100 \%\end{array}$ \\
\hline
\end{tabular}

Fuente: Elaboración según encuesta aplicada.

Tabla 4. Distribución del nivel de las dimensiones de la gestión administrativa

\begin{tabular}{lcccccc}
\hline \multirow{2}{*}{ Dimensiones } & \multirow{2}{*}{ Cantidad } & \multicolumn{6}{c}{ Niveles de dimensiones de la gestión administrativa } & \\
\cline { 2 - 7 } & & Deficiente & Satisfactorio & Competente & Destacado & Total \\
\hline \multirow{2}{*}{ Planeación } & Trabajadores & 1 & 12 & 63 & 10 & 86 \\
& $\%$ & $1 \%$ & $14 \%$ & $73 \%$ & $12 \%$ & $100 \%$ \\
\hline \multirow{2}{*}{ Organización } & Trabajadores & 1 & 21 & 53 & 11 & 86 \\
& $\%$ & $1 \%$ & $24 \%$ & $62 \%$ & $13 \%$ & $100 \%$ \\
\hline \multirow{2}{*}{ Dirección } & Trabajadores & 6 & 22 & 56 & 2 & 86 \\
& $\%$ & $7 \%$ & $26 \%$ & $65 \%$ & $2 \%$ & $100 \%$ \\
\hline \multirow{2}{*}{ Control } & Trabajadores & 8 & 19 & 52 & 7 & 86 \\
& $\%$ & $9 \%$ & $22 \%$ & $61 \%$ & $8 \%$ & $100 \%$ \\
\hline
\end{tabular}

Fuente: Elaboración propia, según encuesta aplicada. 
REVISTA DE LA UNIVERSIDAD DEL ZULIA. 3é época. Año 11 N³1, 2020 José Walter Coronel Chugden et al /// Clima organizacional, satisfacción laboral... 52-70

DOI: $\underline{\text { http://dx.doi.org/10.46925//rdluz.31.05 }}$

En la tabla 4, se analiza el nivel de las 4 dimensiones de la gestión administrativa, por lo que la dimensión planeación presenta un nivel de proporción de: deficiente 1\%, satisfactorio 14\%, competente 73\%, destacado 12\%; para la dimensión organización: deficiente $1 \%$, satisfactorio 24\%, competente 62\%, destacado 13\%; para la dimensión dirección: deficiente 7\%, satisfactorio 26\%, competente 65\%, destacado 2\%; para la dimensión control: deficiente 9\%, satisfactorio 22\%, competente $61 \%$, destacado $8 \%$. Por lo que, se determina que 4 dimensiones, tienen en mayor proporción un nivel competente. Sin embargo, hay una brecha por mejorar, para ello, el orden de prioridad debe darse a la dimensión planeación como prioridad 1, media=3.19 puntos; control deber ser (prioridad 2, media=3.21), dimensión dirección debe ser (prioridad 3, media=3.22), la dimensión organización debe ser (prioridad 4, media=3.51 puntos) y en general la gestión administrativa se tiene un puntaje promedio de 3.39 puntos de un puntaje ideal de 5 puntos.

\section{Tabla 5 .}

Prueba de correlación múltiple de las variables: clima organizacional, satisfacción laboral y gestión administrativa

\begin{tabular}{lc}
\hline \multicolumn{1}{c}{ Estadístico de Prueba } & Valor de prueba \\
\hline Coeficiente de correlación & $0.408^{* *}$ \\
múltiple & 0.001 \\
Significancia P<0.01 & $63.9 \%$ \\
Coeficiente de determinación $\mathrm{R}^{2}$ & $62.1 \%$ \\
$\mathrm{R}^{2}$ ajustado & 7.99 \\
Error típico & 86 \\
Muestra & \\
\hline
\end{tabular}

Fuente: Elaboración según análisis de datos en SPSS, encuesta, P<0.01

En la tabla 5, se analiza la correlación múltiple de las tres variables y se evidencia correlación significativa estadísticamente $(\mathrm{p}<0.01)$. Se puede evidenciar que existe una correlación positiva directa de nivel moderada $\left(\mathrm{Rs}^{=+0.408)}\right.$ entre las variables de estudio: clima organizacional, satisfacción laboral y gestión administrativa.

En la tabla 6, observamos que las correlaciones son significativas estadísticamente p<0.01, por lo que existe una correlación positiva entre variables. Para xl y x2 ( $\mathrm{T}=2.779$, $\mathrm{p}=0.007<0.01)$, para $\mathrm{xl}, \mathrm{x} 3(\mathrm{~T}=2.937, \mathrm{p}=0.004<0.01)$, para $\mathrm{x} 2, \mathrm{x} 3(\mathrm{~T}=3.394, \mathrm{p}=0.001<0.01)$. 
REVISTA DE LA UNIVERSIDAD DEL ZULIA. 3é época. Año 11 N³1, 2020 José Walter Coronel Chugden et al /// Clima organizacional, satisfacción laboral... 52-70

DOI: $\underline{\text { http://dx.doi.org/10.46925//rdluz.31.05 }}$

\section{Tabla 6.}

Prueba $\mathrm{T}$ de significancia de las correlaciones de Spearman de las variables: clima organizacional, satisfacción laboral y gestión administrativa.

\begin{tabular}{lcccc}
\hline \multicolumn{1}{c}{$\begin{array}{c}\text { Prueba de Significancia } \\
\text { de Correlación de variables }\end{array}$} & Rs & $\begin{array}{c}\text { Error } \\
\text { Standar }\end{array}$ & $\begin{array}{c}\text { T } \\
\text { aprox. }\end{array}$ & $\begin{array}{c}\text { Significanci } \\
\text { a }(\mathrm{P}<0.01)\end{array}$ \\
\hline $\begin{array}{l}\text { Clima Organizacional y Satisfacción Laboral (xl y } \\
\text { x2) }\end{array}$ & 0.290 & 0.112 & 2.779 & $0.007^{* *}$ \\
$\begin{array}{l}\text { Clima Organizacional y Gestión Administrativa (xl } \\
\text { y x3) }\end{array}$ & 0.305 & 0.107 & 2.937 & $0.004^{* *}$ \\
$\begin{array}{l}\text { Satisfacción Laboral y Gestión Administrativa (x2 } \\
\text { yx3) }\end{array}$ & 0.347 & 0.107 & 3.394 & $0.001^{* *}$ \\
\hline
\end{tabular}

** Prueba es significativa estadísticamente $\mathrm{P}<0.01$

De igual manera, en figura 2, se presenta la correlación de Spearman bivariado de los puntajes entre cada par de variables del estudio, en la que se evidencia que existe una correlación moderada positiva entre cada par de ellas, por ejemplo para la correlación entre clima organizacional y satisfacción laboral o viceversa( $\mathrm{Rs}=+0.290$ moderada, $\mathrm{p}=0.007<0.01$ ), para la correlación entre el clima organizacional y gestión administrativa o viceversa (Rs=+0.305 moderada, $\mathrm{p}=0.004<0.01$ ), para la correlación satisfacción laboral y gestión administrativa o viceversa $(\mathrm{Rs}=+0.347$ moderada, $\mathrm{p}=0.001<0.01$ ). Lo que se concluye que entre cada par de variables la correlación es positiva directa de nivel moderada y estadísticamente significativa, tal como se evidencia gráficamente:

\section{Discusión}

Los resultados obtenidos en la presente investigación, evidencian que existe una correlación positiva de nivel moderada entre clima organizacional, satisfacción laboral y gestión administrativa (Correlación $\mathrm{R} s=+0.408, \mathrm{p}=0.001<0.05$ ). De igual manera, al evaluar por separado las tres variables de estudio, se encontró que el clima organizacional, mostró ser de nivel alto con una proporción de 79\%; la satisfacción laboral de nivel satisfecho con proporción de 66\% y la gestión administrativa de nivel competente con proporción de 73\%. Tales resultados se comparan con los obtenidos por Hospinal (2013) quien, al investigar la correlación entre el clima organizacional, y satisfacción laboral, encontró que el clima 
REVISTA DE LA UNIVERSIDAD DEL ZULIA. 3é época. Año 11 N³1, 2020 José Walter Coronel Chugden et al /// Clima organizacional, satisfacción laboral... 52-70

DOI: http://dx.doi.org/10.46925//rdluz.31.05

organizacional impacta en un $84.6 \%$ a la satisfacción laboral [pvalue $<0.05$ y el R ${ }^{2}$ es $84.6 \%$.], además, resalta que el clima organizacional y satisfacción laboral son positivos [3.3029, 3.6537], [3.5114, 3.8967]. Sin embargo, encontró factores críticos en la variable clima organizacional: cohesión, equidad y reconocimiento; con importancia de 99.1\%. y en satisfacción laboral: satisfacción por el trabajo en general, satisfacción con el ambiente físico del trabajo, satisfacción con las oportunidades de desarrollo y satisfacción en relación subordinado-jefe, con una importancia de 99.8\%. Resultados que no distan en gran medida comprados a los nuestros.

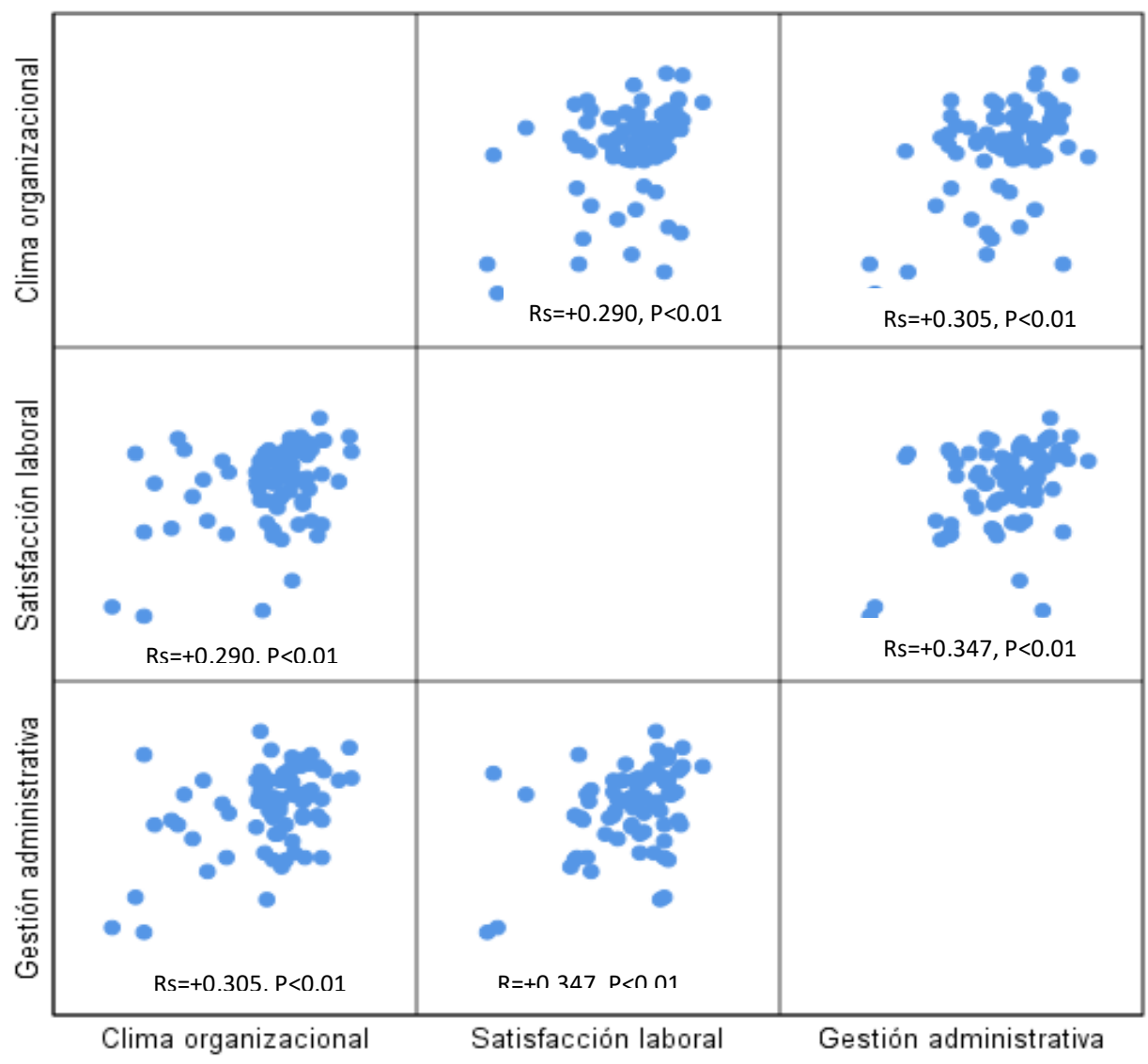

Figura 2. Diagrama de correlación de Spearman bivariado entre las variables de estudio de los puntajes obtenidos de la encuesta aplicada

Similares resultados se muestran en el trabajo realizado por Pecino-Medina et al., (2017), quienes al examinar el efecto mediador de la ambigüedad de rol en la relación entre el clima y la satisfacción laboral en la administración pública local, los resultados mostraron 
REVISTA DE LA UNIVERSIDAD DEL ZULIA. 3é época. Año 11 N³1, 2020 José Walter Coronel Chugden et al /// Clima organizacional, satisfacción laboral... 52-70

DOI: http://dx.doi.org/10.46925//rdluz.31.05

interrelaciones entre las variables $\mathrm{r}=.641(\mathrm{p}<.001)$ y $\mathrm{r}=-.571(\mathrm{p}<.001)$, es decir, existe una relación positiva $(\beta=1,19 ; \mathrm{p}<$. 001), lo cual, permite confirmar la hipótesis de que aumentar o mejorar el clima de los servicios implica una mayor satisfacción laboral de los empleados ( $\beta$ = 1,19; $\mathrm{p}<$. 001). Tales hallazgos coinciden con los obtenidos por nosotros y otras investigaciones realizadas por Pedraza (2018); Fernández-Oliva et al., (2019) y Pecino-Medina et al. (2015).

También se encuentra similitudes con estudios efectuados por Sagredo y Castelló (2019) quienes al correlacionar la gestión directiva y clima organizacional, los resultados arrojaron una correlación de 0,623, lo que es significativo a nivel de 0,01 (bilateral). Dicha valoración estadística señala que la correlación es positiva y significativa, así también al correlacionar las dimensiones gestión directiva con motivación y compromiso docente, encontró una correlación de 0,623 y 0,778 respectivamente.

En línea con lo expuesto anteriormente, también hay semejanzas en la investigación dirigida por Rodríguez et al., (2019) quienes buscaron comprobar la correlación entre clima organizacional y gestión educativa en un Instituto Nacional, encontraron que la correlación es positiva entre las dos variables [Pearson 0,879] , [Valor $\mathrm{p}=0.000<0.05$ ] por lo tanto, concluyeron que el clima organizacional se relaciona significativamente con la gestión educativa y/o administrativa, ante ello precisaron que una correspondencia teórica y práctica entre el clima organizacional y calidad del servicio educativo, ocasiona un mayor desarrollo del clima laboral, mejor satisfacción del estudiante. Asimismo, encontraron una correlación alta en las dimensiones identidad institucional, relaciones interpersonales, dinámica institucional con la calidad del servicio. De manera análoga, ocurre con la investigación de Molina et al., (2019) quienes encontraron que las habilidades administrativas tienen un impacto directamente proporcional sobre las habilidades de gestión.

Finalmente, los resultados obtenidos en una sola medición y al trabajarse únicamente con una Universidad, no pueden ser extrapolados a toda la población, a pesar de ser acopiados con una muestra bastante representativa; en tal sentido, cabría la posibilidad de recoger nuevos datos en diferentes universidades del país, tanto públicas como privadas para realizar comparaciones y tomar decisiones efectivas sobre los mismos. 
REVISTA DE LA UNIVERSIDAD DEL ZULIA. 3é época. Año 11 N³1, 2020 José Walter Coronel Chugden et al /// Clima organizacional, satisfacción laboral... 52-70

DOI: $\underline{\text { http://dx.doi.org/10.46925//rdluz.31.05 }}$

Conclusiones

Después de ejecutada la investigación, contrastadas y comprobada la hipótesis de investigación $\left(\mathrm{H}_{1}\right)$ se llegó a las conclusiones siguientes: existe una correlación positiva de nivel moderada [(Correlación $\mathrm{Rs}=+0.408, \mathrm{p}=0.001<0.05)$ entre el clima organizacional, satisfacción laboral y gestión administrativa. Así también, el clima organizacional es de nivel alto (proporción=79\%), la satisfacción laboral es de nivel satisfecho (proporción =66\%) y la gestión administrativa es de nivel competente (proporción=73\%). También se concluye, que existe una correlación moderada positiva significativamente entre par de variables, clima organizacional y satisfacción laboral ( $\mathrm{Rs}=+0.290$ moderada, $\mathrm{p}=0.007<0.01$ ) clima organizacional y gestión administrativa ( $\mathrm{Rs}=+0.305$ moderada, $\mathrm{p}=0.004<0.01$ ) satisfacción laboral y gestión administrativa ( $\mathrm{Rs}=+0.347$ moderada, $\mathrm{p}=0.001<0.01)$.

\section{Referencias}

Adeniji, A., Salau, O., Awe, K., \& Oludayo, O. (2018). Survey datasets on organisational climate and job satisfaction among academic staff in some selected private universities in Southwest Nigeria. Data in Brief, 19, 1688-1693. https://doi.org/10.1016/j.dib.2018.06.001

Aguirre, C., Vauro, M., Labarthe, J., \& Vauro, R. (2015). Estresores laborales y bienestar en el trabajo en personal aeronáutico de cabina. Ciencias Psicológicas, 9(2), 292-308. https://doi.org/10.22235/cp.v9i2.616

Azanza, G., Moriano, J. A., \& Melero, F. (2015). Estrés de rol y satisfacción laboral: examinando el papel mediador del engagement en el trabajo. Journal of Work and Organizational Psychology, 31(2), 69-77. https://doi.org/http://dx.doi.org/10.1016/j.rpto.2015.04.001

Bernal, I., Angélica, N., Melo, P., Lorena, M., \& Limón, S. (2015). El clima organizacional y su relación con la calidad de los servicios públicos de la salud: diseño de un modelo teórico. In Estudios Gerenciales (Vol. 31, Issue 134, pp. 8-19). https://doi.org/10.1016/j.estger.2014.08.003

Bustamante-Ubilla, M. A., Lissette, M., Avenda, G., \& Maza, L. (2015). Caracterización del clima organizacional en hospitales de alta complejidad en Chile. Estudios Gerenciales, 31, 432440. https://doi.org/http://dx.doi.org/10.1016/j.estger.2015.08.003

Cajas, M., Gastiaburo, J., Espinel, J., \& R Ruperti, E. (2017). Factores de satisfacción e insatisfacción laboral en trabajadores de la ciudad de milagro. Revista de Psicología UNEMI, 1(2602-8379), 15-23.

Castillón, A. M. (2014). Fundamentos generales de administración. Centro Editorial Esumer. 
REVISTA DE LA UNIVERSIDAD DEL ZULIA. 3é época. Año 11 N³1, 2020 José Walter Coronel Chugden et al /// Clima organizacional, satisfacción laboral... 52-70

DOI: $\underline{\text { http://dx.doi.org/10.46925//rdluz.31.05 }}$

Díaz, G., Peña, carolina M., \& Castellanos, B. A. (2014). El liderazgo y las relaciones interpersonales dentro del clima organizacional. Revista Global de Negocios, 2(2010), 1-11.

Espinoza, P., Peduzzi, M., Agreli, H. F., \& Sutherland, M. A. (2018). Interprofessional team member's satisfaction: A mixed methods study of a Chilean hospital. Human Resources for Health, 16(1), 1-12. https://doi.org/10.1186/s12960-018-0290-z

Falkenström, F., Grant, J., \& Holmqvist, R. (2018). Review of organizational effects on the outcome of mental health treatments. Psychotherapy Research, 28(1), 76-90. https://doi.org/10.1080/10503307.2016.1158883

Galicia, D., \& Monroy, M. E. (2016). La retórica y el discurso administrativo. Contaduria y Administracion, 6l(3), 582-598. https://doi.org/10.1016/j.cya.2015.06.003

García-Guiu, C., Molero, F., \&e Moriano, J. A. (2014). El liderazgo auténtico y su influencia sobre la cohesión grupal y la identificación organizacional: El papel de la justicia organizacional como variable mediadora. Revista de Psicologia Social, 30(1), 60-88. https://doi.org/10.1080/02134748.2014.987539

Gómez-Perdomo, G. E., Meneses-Higuita, A. C., \& Palacio-Montes, M. C. (2017). Job satisfaction and psychological capital: Factors that affect burnout syndrome. Ansiedad $y$ Estres, 23(2-3), 71-75. https://doi.org/10.1016/j.anyes.2017.09.002

Gónzales, M. B., Figueroa, E. G., \& Gonzáles, R. C. (2014). Influencia del liderazgo sobre el clima organizacional en PYMES: caso cetering Gourmet de Durango, México. Suma de Negocios, 5(11), 117-125. https://doi.org/10.1016/s2215-910x(14)70026-6

Hernández, H. G., Cardona, D. A., \& Del Rio, J. L. (2017). Direccionamiento estratégico: Proyección de la innovación tecnológica y gestión administrativa en las pequeñas empresas. Informacion Tecnologica, 28(5), 15-22. https://doi.org/10.4067/S0718-07642017000500003

Hernández, J., Espinoza, J. de J., \& Aguilar, M. (2013). Clima laboral: la influencia de la supervisión, los factores organizacionales y la predisposición de los empleados. Revista Internacional Administracipon \& Finazas, 6(6), 55-71.

Hospinal, S. (2013). Clima organizacional y satisfacción laboral en la empresa F y D inversiones S.A.C. 16(2), 2103. https://doi.org/https://doi.org/10.15381/idata.vl6i2.11924

Kohantorabi, F., \& Abolmaali, K. (2014). The Prediction of Job Satisfaction Based on Coping Skills in Pilots and Assistant of Pilots. Journal of Service Science and Management, 07(03), 260-266. https://doi.org/10.4236/jssm.2014.73023

Lima, J. L., Bezerra, R. S., Santos, F. Dos, Costa, R. P., \& Reis, L. (2013). Estressores na atividade gerencial do enfermeiro: implicações para saúde. Avances En Enfermería, 31(2), 144152. 
REVISTA DE LA UNIVERSIDAD DEL ZULIA. 3é época. Año 11 N³1, 2020 José Walter Coronel Chugden et al /// Clima organizacional, satisfacción laboral... 52-70

DOI: http://dx.doi.org/10.46925//rdluz.31.05

Magallán, N. (2018). "El clima organizacional y su influencia en el desempeño laboral de los docentes de la Facultad de Ciencias Económicas y Administrativas de la Universidad Nacional Toribio Rodríguez de Mendoza de Amazonas, Chachapoyas 2017-II" [Tesis de pregrado, Universidad Nacional Toribio Rodríguez de Mendoza]. http://cort.as/-IdFS

Manosalvas, C. A., Manosalvas, L. O., \& Nieves, J. (2015). El clima organizacional y la satisfacción laboral: un análisis cuantitativo riguroso de su relación. AD-Minister, 26, 5-15. https://doi.org/10.17230/ad-minister.26.1

Martínez-Corona, J. I., Palacios-Almón, G. E., \& Juárez-Hernández, L. G. (2020). Análisis de validez de constructo del instrumento: "Enfoque Directivo en la Gestión para Resultados en la Sociedad del Conocimiento. Retos, 10(19), 153-165. https://doi.org/10.17163/ret.n19.2020.09

Mendoza Deza, Yris Adriana; Vásquez Gastelumendi, Dora Victoria; Ríos Campos, Carlos Alberto; Camacho Delgado, Freddy Manuel; Gutiérrez Valverde; Karina Silvana (2020). Estrategias motivacionales para mejorar las relaciones interpersonales en los docentes de la Escuela Profesional de Tecnología Médica de la Universidad de Chiclayo, Revista de la Universidad del Zulia, 11 (30), 415-437. DOI: http://dx.doi.org/10.46925//rdluz.30.26

Menoza-Briones, A. (2017). Importancia de la gestión administrativa para la innovación de las medianas empresa comerciales en la ciudad de Manta. Revista Cientiúica Las Ciencias, 3(2), 947-964. https://doi.org/http://dx.doi.org/10.23857/dom.cien.pocaip.2017.3.2.may. 947-964

Merino-Plaza, M. J., Carrera-Hueso, F. J., Roca-Castelló, M. R., Morro-Martín, M. D., Martínez-Asensi, A., \& Fikri-Benbrahim, N. (2018). Relación entre la satisfacción laboral y la cultura de seguridad del paciente. Gaceta Sanitaria, 32(4), 352-36l. https://doi.org/10.1016/j.gaceta.2017.02.009

Molina, L. A., Piñón, L. C., \& Gallegos, V. M. (2019). Análisis de las habilidades administrativas y de gestión en las micro y pequeñas empresas de la ciudad de Chihuahua. Nova Scientia, 11(1), 293-322. https://doi.org/doi.org/10.21640/ns.vlli22.1751

Navarro, A. (2012). Clima organizacional y satisfacción laboral en la empresa actual. RECERCAT (Dipòsit de La Recerca de Catalunya), 16(2), 1-89. http://recercat.cat/handle/2072/203748\%0Ahttp://www.recercat.cat/handle/2072/203748

Onofre, R., Aguirre, C., \& Murillo, K. (2017). Importancia de la gestión administrativa para la innovación de las medianas empresa comerciales en la ciudad de Manta. La Cultura Tributaria y Su Incidencia En La Recaudación de Los Tributos En El Cantón Babahoyo, Provincia de Los Ríos, 3, 3-23. file://C:/Users/Windows/Downloads/DialnetLaCulturaTributariaYSuIncidenciaEnLaRecaudacionDeL-6326643 (1).pdf

Ortiz, D. A. C., Salgado, P. M., \& Cázares, F. L. (2017). Common method variance in the relationship between job satisfaction and life satisfaction. Estudios Gerenciales, 33(145), 321329. https://doi.org/10.1016/j.estger.2017.11.004 
REVISTA DE LA UNIVERSIDAD DEL ZULIA. 3é época. Año 11 N³1, 2020 José Walter Coronel Chugden et al /// Clima organizacional, satisfacción laboral... 52-70

DOI: $\underline{\text { http://dx.doi.org/10.46925//rdluz.31.05 }}$

Palma, L. M. (2016). Modernización judicial, gestión y administración en América Latina. Acta Sociológica, 72, 149-203. https://doi.org/10.1016/j.acso.2017.06.007

Pecino-Medina, V., Díaz-Fúnez, P. A., \& Mañas-Rodríguez, M. A. (2017). Climate, stress and satisfaction: a multilevel study in the public sector / Clima, estrés y satisfacción: un estudio multinivel en el sector público. Revista de Psicologia Social, 32(1), 52-79. https://doi.org/10.1080/02134748.2016.1248027

Pecino-Medina, V., Mañas-Rodríguez, M. A., Díaz-Fúnez, P. A., \& López-Puga, J. (2015). Clima y satisfacción laboral en el contexto universitario. Anales de Psicología, 31(2), 658-666. https://doi.org/http://dx.doi.org/10.6018/analesps.31.2.171721

Pedraza, N. A. (2018). El clima organizacional y su relación con la satisfacción laboral desde la percepción del capital humano. Revista Lasallista de Investigación, 15(1), 90-101. https://doi.org/10.22507/rli.v15nla9

Peña, M. C., Díaz, G., \&e Carillo, A. (2015). Relación del clima organizacional y la satisfacción laboral en una pequeña empresa familiar. 8(1985), 37-50. https://bit.ly/2IyhjUa

Plascencia-Campos, A. R., Pozos-Radillo, B. E., Preciado-Serrano, M. de L., \& Vázquez-Goñi, J. M. (2019). Effect of cognitive-emotional training on job satisfaction and burnout on nursing staff: A pilot study. Ansiedad y Estres, 25(1), 35-41. https://doi.org/10.1016/j.anyes.2019.04.002

Riffo, R. S. (2019). Gestión administrativa y de calidad en los centros escolares de los Chorrillos. Revista Scientific, $\quad 4, \quad 153-172$. https://doi.org/https://doi.org/10.29394/Scientific.issn.2542-2987.2019.4.E.9.153-172

Rodríguez, L., Santos, O., Macazana, M. D., \& Talavera, Y. (2019). Clima organizacional y gestión administrativa del Instituto Nacional de Danza Raymond Mauge Thoniel Guayaquil-Ecuador. 4(2), 5259. https://doi.org/https://doi.org/10.37292/riccva.v4i2.150

Román-Calderón, J. P., Krikorian, A., Franco Ruiz, C., \& Betancur Gaviria, A. (2016). Apoyo organizacional y empoderamiento como antecedentes de comportamientos empoderados y participación de los empleados. Estudios Gerenciales, 32(139), 154-161. https://doi.org/10.1016/j.estger.2016.03.002

Sagredo, E., \& Castelló, A. (2019). Gestión directiva y clima organizacional en la educación de personas adultas en Chile. Actualidades Investigativas En Educación, 19(2), 1-23. https://doi.org/10.15517/aie.v19i2.36895

Sánchez, H., \& Reyes, C. (2017). Metodología y diseños en la investigación científica. Bissines Support Aneth S.R.l.

Santillán, R. M. (2015). Clima organizacional y nivel de satisfacción en la municipalidad provincial de la forntera de los chachapoyas, región Amazonas-2015 [Tesis de pregrado, Universidad Nacional Toribio Rodríguez de Mendoza]. https://bit.ly/2KgJDMF 
REVISTA DE LA UNIVERSIDAD DEL ZULIA. 3e época. Año 11 N³1, 2020 José Walter Coronel Chugden et al /// Clima organizacional, satisfacción laboral... 52-70

DOI: http://dx.doi.org/10.46925//rdluz.31.05

Terrones, R. A. (2017). Compromiso organizacional y su influencia en la satisfacción laboral de la empresa de transportes S.A.C-Agencia Chachapoyas, 2016 [Tesis de pregrado, Universidad Nacional toribio Rodríguez de Mendoza]. https://bit.ly/3gAcCJU

Tinoco, O., Quispe, C., \& Beltrán, V. (2014). Cultura organizacional y satisfacción laboral en la facultad de Ingeniería Industrial en el marco de la acreditación universitaria. Industrial Data, 17(2), 56. https://doi.org/10.15381/idata.v17i2.12048

Vallellano, M. D., \& Rubio-Valdehita, S. (2018). Mental workload and job satisfaction: a comparative study amongst social workers, social educators and primary school teachers. Ansiedad y Estres, 24(2-3), 119-124. https://doi.org/10.1016/j.anyes.2018.08.003

Weiss, H. M. (2002). Deconstructing job satisfaction. Separating evaluations, beliefs and affective experiences. Human Resource Management Review, 12(2), 173-194. https://doi.org/10.1016/S1053-4822(02)00045-1

Yew, S. Y., Yong, C. C., Tey, N. P., Cheong, K. C., \& Ng, S. T. (2018). Work satisfaction among nurses in a private hospital. International Journal of Healthcare Management, 0(0), 1-8. https://doi.org/10.1080/20479700.2018.1489459 